\title{
TRAITEMENTS THERMIQUES ET VALEUR ALIMENTAIRE
}

\author{
PAR \\ RAYMOND JACQUOT \\ Laboratoire de Biochimie de la Nutrition du C. N. R. S., Bellevue.
}

\section{Observations liminaires $\left({ }^{1}\right)$}

Les indications du thermomètre ne sont qu'une des caractéristiques d'un traitement thermique donné. Plusieurs autres facteurs sont aussi importants que la température, à savoir la durée du chauffage, les conditions de transmission de la chaleur, la conductibilité thermique du produit chauffé et même sa composition chimique.

Pour être strictement objective, notre présente étude devrait préciser toutes les modalités qui accompagnent la cuisson des aliments. Cela n'a pas toujours été possible en raison de l'imprécision de certaines données fournies par la littérature. Du moins pouvons-nous dégager les grandes lignes du problème et indiquer le sens de l'effet thermique sur les principaux aliments. Mais avant il convient de rappeler quels sont les métabolites à caractère thermolabile.

\section{Les vitamines et les antivitamines}

On a longtemps accusé la cuisson des aliments - et à plus forte raison les modes de chauffage industriel - de "tuer" les vitamines. Or l'expérience montre toute l'exagération d'une telle créance. Voici, en effet, un tableau emprunté à CLIFCORN qui fait le point de la question.

\footnotetext{
Vitamines

Carotène, Vitamine $A \ldots \ldots \ldots \ldots \ldots \ldots$

Thiamine $\ldots \ldots \ldots \ldots \ldots \ldots \ldots \ldots \ldots \ldots$

Riboflavine ...................

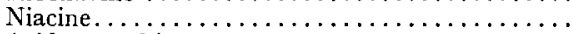

Acide ascorbique $\ldots \ldots \ldots \ldots \ldots \ldots \ldots \ldots \ldots \ldots \ldots \ldots$

Vitamines
}

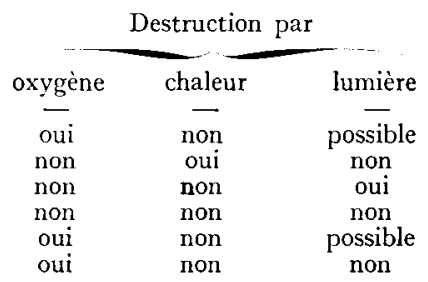

(1) Ce texte fait de larges emprunts au travail précédemment publié avec la collaboration de J. Matet et de O. Fridenson (voir bibliographie). 
Si nous ajoutons que tous les membres du complexe B2, à savoir pyridoxine, acides pantothénique et folique, vitamine Br2 et Animal Protein Factor, biotine etc..., sont réputés thermostables, on conviendra que la sensibilité à la chaleur des vitamines tient de la légende.

En fait, seule la vitamine BI est à coup sûr altérée par chauffage. Mais sa destruction dépend de la nature des traitements thermiques. Alors que la friture entraîne une complète disparition, la cuisson à l'eau ne détermine qu'un léger déficit; la cuisson des petits pois ne fait perdre que 4 p. Ioo de thiamine, mais $37 \mathrm{p}$. Ioo passent dans l'ean de cuisson.

Cependant, il faut reconnaître que l'application de chaleur industrielle peut s'accompagner de phénomènes secondaires mettant en jeu l'oxygène ou la lumière et entraîner ainsi des pertes vitaminiques. Il conviendrait donc d'étudier systématiquement la conservation des vitamines dans tous les produits manufacturés. I,e problème a été minutieusement étudié en ce qui concerne des denrées utilisées en alimentation humaine : conserves animales ou végétales, jus de fruits, confiture etc. Ce point ne nous intéresse pas directement. Les données relatives aux produits susceptibles d'être consommés par le bétail sont, par contre, plus rares et pas toujours concordantes.

La dessication artificielle des fourrages conduit à une meilleure tenue de la vitamine A (carotène) que le séchage naturel. Voici des chiffres publiés par ZIMMERMANN :

$\begin{array}{cc}\begin{array}{c}\text { Carotène } \\ \text { en } \mathrm{mg} / \mathrm{r} \text { oog secs du matériel de base } \\ \hline 60\end{array} & - \\ 45 & -25 \\ 5 \mathrm{I} & -\mathrm{I} 5 \\ 55 & -8\end{array}$

Herbe fraîche (matériel de base) ..............

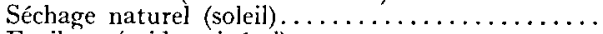

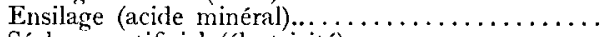

Séchage artificiel (électricité) $\ldots \ldots \ldots \ldots \ldots \ldots \ldots$

I,es céréales font souvent 1'objet de traitements spéciaux dans le but d'obtenir des produits biscuités, gonflés ou expansés. Comme ceux-ci sont susceptibles d'être utilisés dans le régime des veaux ou de la volaille, il est bon de connaitre leur potentiel vitaminique. Alors qu'en boulange la destruction de thiamine est faible et peut ne pas dépasser 7 p. IOo (HARREL et col.), elle est beaucoup plus intense lors du biscuitage ou de l'expansion. Dans le premier cas, un traitement rapide (7o secondes) détermine des pertes de l'ordre de 20 à 30 p. IOO (HARREL et col.). Quant à l'expansion, elle est réellement meurtrière pour la vitamine BI qui disparait en totalité (Bоотн et col.). Il est juste cependant de remarquer qu'elle respecte les facteurs du groupe $\mathrm{B} 2$, ce qui confirme leur réelle thermostabilité.

Les différents traitements du lait peuvent également retentir sur la teneur en vitamines. La pasteurisation ne touche en rien les vitamines $\mathrm{A}, \mathrm{B}$ ou $\mathrm{D}$. La seule perte significative intéresse la vitamine $\mathrm{C}$ dont le taux peut être diminué de $20 \mathrm{p}$. Ioo. Encore cette destruction est-elle d'ordie photochimique (KON). La concentration a des effets plus graves 
et détruit parfois $40 \mathrm{p}$. roo de la vitamine $\mathrm{BI} ; 1$ 'évaporation sur cylindre est encore plus préjudiciable et la moitié de la thiamine risque ainsi de disparaître. Il convient d'ajouter que l'atomisation constitue de ce point de vue un réel progrès.

L,es levures de brasserie ou de distillerie représentent un précieux aliment pour le bétail. Il est vraisemblable que leur dessication entrầne une perte de thiamine. Mais il s'agit là d'un mal nécessaire, car les levures ne sont pas alimentaires à 1'état frais. Non seulement, elles ne sont pas digérées sous cette forme, mais peuvent soit causer des accidents dûs à leur pouvoir fermentaire, soit agir comme facteur antivitaminique par suite d'une compétition entre leurs besoins propres (qui continuent à se manifester au cours du transit intestinal) et les besoins de 1'hôte.

Fin conclusion de cette courte revue, nous voyons que les traitements thermiques ne sont réellement préjudiciables qu'à la thiamine. Or cette vitamine n'a de signification nutritionnelle que pour le Porc et la Volaille. Certes, sa destruction représente ici une diminution du pouvoir alimentaire, mais elle est beaucoup moins grave que ne serait celle du carotène, qui demeure la vitamine majeure tant pour les monogastriques que pour les ruminants.

Pour compléter notre information et faire le bilan des avantages et défauts des traitements thermiques vis-à-vis des vitamines, il faut mentionner la thiaminase. Cette antivitamine se trouve dans certains poissons et peut être même dans certaines viandes. Il ne s'agit pas là d'une curiosité de laboratoire puisque sa découverte a été provoquée par les accidents causés dans un certain type d'élevage, celui du renard à fourrure. La thiaminase constitue un véritable facteur de malnutrition. Or elle est très sensible à l'action de la chaleur $\left(^{1}\right)$; on peut considérer la cuisson $\mathrm{du}$ poisson comme une opération scientifiquement basée. Cette notion ne devrait pas être oubliée en ce moment, où de très nombreux industriels s'ingénient à réaliser des autolysats de poisson à partir de viscères crues. La thiaminase est particulièrement abondante dans le tractus gastro-intestinal et ses glandes annexes. On peut dès lors se demander si de tels autolysats ne présentent pas une action antivitaminique et s'il ne serait pas bon de leur faire subir un traitement thermique.

\section{Autres principes thermolabiles}

Sous cette rubrique, on peut tout d'abord classer un bon nombre de glucosides toxiques. Un traitement thermique est obligatoire pour les détruire ou en permettre l'extraction. Le manioc, le lin, certains $P h a-$ seolus peuvent contenir des glucosides générateurs d'acide cyanhydrique.

(1) Il est juste de signaler l'existence de thiaminases végétales à caractère thermorésistant, comme les antivitamines de la fougère et des céréales. 
Cet inconvénient disparaît après ébullition ou tout autre application de chaleur. Le colza renferme des glucosides générateurs d'isosulfocyanate d'allyle et de crotonine. Pour détoxiquer ce tourteau, il faut entraîner par la vapeur les sénévols volatils, donc procéder à une cuisson. Le coton est réputé contenir du gossypol, mais ce toxique est en grande partie éliminé au cours de l'extraction de l'huile et le d-gossypol des farines de coton délipidées ne présente pas de danger. Nous pourrions aussi mentionner l'exemple de 1'hémolysine, également détruite par le chauffage. Mais nous ne saurions nous attarder sur ce problème essentiellement toxicologique.

Plus réellement alimentaire est le problème du phosphore phytique. Les phytates, sont très largement représentés dans le monde végétal et particulièrement dans les graines à aleurone. L'acide phytique agit comme un déminéralisant en insolubilisant le calcium et le fer des aliments. Sa présence dans la ration peut donc réaliser des états carentiels, tout au moins chez les monogastriques. A vrai dire le mécanisme anticalcifiant de l'acide phytique est encore bien obscur, comme le souligne une revue récente de GuILLEMET, Jacouot et Trémolik̀res. Mais l'important est que les traitements thermiques soient susceptibles d'hydro1yser l'acide phytique en inositol et acide phosphorique, empêchant ainsi la formation de phytates insolubles. C'est ainsi qu'on ne trouve plus trace d'acide phytique dans le blé expansé (Bootr et col.).

Sur le plan général, on voit qu'il existe, parmi les principes thermolabiles, des métabolites efficaces et des facteurs de mal-nutrition; c'est dire que les inconvénients du chauffage le disputent aux avantages. Ce bilan ainsi dressé, nous pouvons aborder l'influence propre des traitements thermiques sur les principaux aliments.

\section{Les pommes de terre}

Ces tubercules constituent avant tout un aliment énergétique au même titre que le manioc. Mais alors que l'amidon de manioc est parfaitement digestible à l'état cru, la fécule exige une cuisson pour être bien digérée. Des mesures faites sur le Rat permettent d'attribuer un CUD de moins de 50 à la fécule crue contre 98 au produit cuit. Ces différences se retrouvent chez le Porc; en outre la fécule crue provoque de graves accidents intestinaux. Il y a donc un avantage certain à utiliser la pomme de terre sous la forme de farine autoclavée (flocons). La résistance cux sucs digestifs de la fécule crue est difficilement interprétable. On l'explique cependant en invoquant une trop haute polymérisation que réduit la cuisson aussi bien que 1'ultra-mouture (SUTRA ; LEVY et JACQUO'T).

Cet exemple est le seul que nous puissions citer quant aux effets de 1a chaleur sur 1'utilisation des hydrates de carbone. En effet, dans tous 
les autres cas, les traitements thermiques ne semblent pas influencer dans un sens quelconque les aptitudes alimentaires des sucres ou des amidons. Cela semble certain pour la chaleur humide et probable pour la chaleur sèche. Nous n'en possédons pas la preuve expérimentale, mais il paraît vraisemblable que les caramels sont aussi "énergétiques " que les sucres dont ils dérivent. Nous disons caramels et non charbon, car tout excès thermique conduit à la carbonisation, donc à la perte du pouvoir alimentaire.

Tout ce qui va suivre concernera donc l'influence (très paradoxale) de traitements thermiques sur l'efficacité protidique.

\section{Les farines de tissus animaux}

I,es répercussions des traitements industriels subis par les farines de poisson ont été passées en revue par CREAC'H lors du dernier Congrès du Poisson (Paris I950). Nous croyons que ses conclusions peuvent également intéresser les farines de viande et de sang.

Tout un ensemble d'expérimentateurs a démontré la sensibilité thermique des protides du poisson. Pour des farines de même origine la hiéarchie de l'efficacité protidique et de la digestibilité peut s'établir schématiquement dans 1'ordre décroissant suivant :

Gain pondéral du Rat

(g/g de nourriture)

Farine séchée sous vide $\ldots \ldots \ldots \ldots \ldots \ldots \ldots \ldots \ldots$

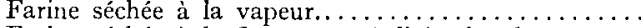

0,27

0,22

Farine séchée à la flamme ou à l'air chaud.........

0,16

On sait d'autre part que les farines peuvent être préparées par voie sèche ou par voie humide (avec cuisson préalable et pression des tissus). Il semble que 1'azote des farines obtenues par voie humide soit plus efficacement utilisé que celui des produits de même origine obtenus par voie sèche. Voici un exemple portant sur la farine de déchets de Haddock:

\begin{tabular}{|c|c|}
\hline Traitement & CUD \\
\hline Séchage : & - \\
\hline $\begin{array}{l}\text { à la flamme... } \\
\text { à la vapeur... } \\
\text { sous vide .... }\end{array}$ & $\begin{array}{l}80 \\
93 \\
96\end{array}$ \\
\hline Cuisson et séchag & \\
\hline $\begin{array}{l}\text { à la flamme... } \\
\text { à la vapeur... } \\
\text { sous vide .... }\end{array}$ & $\begin{array}{l}86 \\
93 \\
96\end{array}$ \\
\hline
\end{tabular}

A première vue, on pourrait s'étonner de ce que la cuisson améliore l'efficacité des protides du poisson, alors que les traitements thermiques ultérieurs s'avèrent d'autant plus nocifs qu'ils sont plus intenses. Y at-il dans ce cas une sorte de dualité dans les effets du chauffage : bénéfice jusqu'à un certain seuil de température, perte au delà ? Il n'en est rien, 
car les différences observées in vivo entre farines par voie sèche ou par voie humide ne sont pas imputables à la cuisson, mais au pressage.

Dans les farines de déchets préparées par voie humide le pourcentage d'azote extractif n'est que de Io à I 5 p. roo, au lieu de 20 à 25 p Ioo. dans les produits préparés par voie sèche. Le passage à la presse débarrasse la farine d'une importante fraction d'azote soluble. Or, cette forme d'azote possède une efficacité protidique inférieure à celle de l'azote insoluble: la VB est en effet de 34 pour les formes azotées solubles, contre 97 pour l'azote fixe. Autrement dit la technique par voie humide valorise le produit final par élimination d'azote "inférieur». Ce raisonnement se défend, mais il n'en reste pas moins que la cuisson et le pressage entraînent une perte sèche d'azote et de vitamines, tout particulièrement en facteurs de croissance du groupe Animal Protein Factor. La méthode dite par voie humide comporte donc des avantages et des inconvénients. Nous ne sommes pas en mesure d'en adresser le bilan exact.

Dans la pratique, la sensibilité thermique des protides du poisson a des répercussions certaines et peut, à elle seule, expliquer les différences de rendement entre deux produits de même origine. Voici un exemple emprunté à MANnING et HoNCAMP et qui intéresse le Porc :

\begin{tabular}{|c|c|}
\hline & $\begin{array}{l}\text { Gain pondéral } \\
\text { journalier }(\mathrm{g})\end{array}$ \\
\hline Farine de White Fish séchée à la flamme $\ldots \ldots \ldots \ldots \ldots$ & 763 \\
\hline Farine de White Fish séchée sous vide $\ldots \ldots \ldots \ldots \ldots \ldots$. & 927 \\
\hline 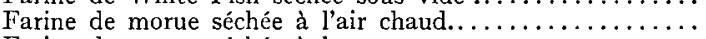 & 665 \\
\hline Farine de morue séchée à la vapeur ............... & 672 \\
\hline
\end{tabular}

Une conclusion s'impose donc: pour obtenir une bonne farine de poisson dont la fraction protidique aura perdu le minimum de qualités, il sera toujours indiqué :

D'opérer à température aussi basse que possible. L'expérience montre que les températures comprises entre 85 et $105^{\circ}$ constituent des points critiques à ne pas dépasser selon l'origine et la nature du matériel traité;

D'écourter le plus possible les temps de chauffe.

La perte d'efficacité protidique qu'entrainent les traitements thermiques excessifs s'expliquent, dans le cas du poisson comme dans celui de nombreux autres aliments, par une baisse simultannée du CUD et de la VB (voir le tableau précédent).

Que des opérations, qui modifient profondément la nature physicochimique des protides, interviennent dans les processus de 1'attaque digestive, rien n'est plus facilement concevable. Mais ce qui l'est moins, c'est que l'action de la chaleur, tantôt facilite l'hydrolyse enzymatique, tantôt lui nuit. Car, par la suite nous verrons des cas où les traitements thermiques améliorent la digestibilité, alors qu'en général ils la diminuent. Aussi est-on réduit à admettre que, selon la nature des protides, la dénaturation thermique entrave ou favorise l'utilisation digestive. Cela n'ex- 
plique rien. Quoi qu'il en soit, les protides du poisson semblent d'autant mieux digérés qu'ils ont été moins cuits.

Quant à la Valeur Biologique, elle traduit en quelque sorte 1'équilibre des aminoacides. Il faudrait donc admettre que le chauffage détruit certains essentiels dans les protides du poisson. Nous discuterons cette hypothèse à propos dı lait. Disons de suite qu'elle n'est pas fondée : il n'y a pas disparition, mais indisponibilité, ce que les Anglo-Saxons appellent "inavailability ". Dans le poisson, c'est la lysine qui est en cause: au cours du chauffage il se développe, soit une forme résistante aux enzymes, soit des anomalies dans les liaisons peptidiques. Il en résulte que le poisson chauffé apparaît biologiquement comme déficient en lysine et cette déficience explique parfaitement la baisse de la VB. Cette hypothèse reçoit confirmation du fait que l'extra-lysine supplémente beaucoup plus efficacement une farine de poisson surcuite qu'un produit préparé à basse température. D'ailleurs, nous verrons que l'altération de la lysine au cours du chauffage des protéines n'est pas un caractère spécifique des farines de poisson, mais un phénomène d'une portée générale.

\section{Les produits laitiers}

Il est certain que l'efficacité protidique du lait est diminuée par chauffage. C'est ainsi que l'autoclavage inhibe les aptitudes à la croissance, qu'il s'agisse de la poudre de lait (VoEgTLIN et Thompson), de la caséine (Mc Collum avec le Rat ; Gerlung avec la Souris) et de la lactalbumine (Chick et col.). Ces derniers auteurs ont d'ailleurs démontré que la chaleur sèche semblait moins nocive que la chaleur humide.

Cette sensibilité n'a cependant pas de grosses répercussions dans la pratique alimentaire. Il n'y a, en effet, aucune commune mesure entre les traitements thermiques réalisés au laboratoire et ceux en usage dans 1'Industrie Laitière: dans le premier cas on fait appel à des cuissons prolongées : 72 heures à I I $2^{\circ}$ ou 66 heures à $150^{\circ}$ en atmosphère sèche ; autoclavage d'au moins I heure. Toute autre est la technique industrielle : dans le séchage sur cylindres (Roller Process) la température du lait ne dépasse pas I Io à $I 20^{\circ}$ et cela pendant quelques secondes; dans l'atomisation (Spray Process) le brouillard de lait est immédiatement séché. Seule la concentration peut comporter des opérations relativement longues. Donc, a priori, les laits industriels semblent protégés contre toute altération d'origine thermique.

C'est ce que prouve l'expérience. HENRY et col., comparant 3 types de laits (concentré, atomisé, séché sur cylindres) préparés à partir d'un même lait frais, aboutissent aux conclusions suivantes:

- les deux laits secs ont une même digestibilité, par contre celleci est abaissée pour le lait concentré ; 
- la VB des 3 échantillons est identique ;

- la valeur de croissance pour le Rat est identique dans tous les cas. On admet que la dessication industrielle du lait n'est préjudiciable qu'en cas d'opération mal conduite. Dans un lait surchauffé au cours du traitement sur cylindres, FAIRBANKs et Mitcheis ont, en effet, noté une baisse de $1 \mathrm{~V}$ VB atteignant $30 \%$.

Bien que le sujet sorte du cadre de cette étude, il faut parler ici de l'importance du facteur température, non plus au cours du traitement du lait, mais pendant la conservation des laits secs. Des échantillons entreposés à $3^{6-47^{\circ}}$ pendant 60 jours accusent une diminution considérable de 1'efficacité de croissance qui diminue de près de $50 \%$ et de la VB qui tombe de 85 à 65 .

L'ensemble des faits conduit aux conclusions suivantes :

Les traitements thermiques n'améliorent jamais l'efficacité des protides du lait; un chauffage intense l'altère considérablement; cependant les techniques industrielles bien conduites ne présentent aucunement cet inconvénient.

La température de conservation du lait sec a une grande importance et un entreposage à $40^{\circ}$ diminue fortement sa valeur protidique. A noter que cette altération s'accompagne d'une perte de solubilité et de modifications dans la répartition de l'azote et le taux des sucres des cénapses.

Bien que sans répercussions dans le domaine pratique, la sensibilité thermique des protides du lait pose un intéressant problème théorique qui a été particulièrement bien étudié. Nous en décrivons l'évolution qui pourra servir également de démonstration dans d'autres cas.

Dans ses essais d'autoclavage de la caséine, Miss CHICK observe que la perte d'efficacité peut s'expliquer à la fois par une chute du CUD (de 93 à 73) et une baisse de la VB (de 64 à 44).

Nous avons dit comment il fallait interpréter la diminution de digestibilité : dénaturation des protides et inhibition partielle de l'attaque enzymatique. En ce qui regarde la VB, voici comment on est arrivé à la notion d'indisponibilité d'un aminoacide indispensable.

On peut restituer à la caséine chauffée son efficacité première en la supplémentant avec de l'extra-lysine (GREAVEs). Il était alors naturel de penser que le chauffage détruisait la lysine au même titre que 1'hydrolyse acide détruit le tryptophane. Il n'en est rien car, par méthode chimique, on trouve la même quantité de lysine dans la caséine crue ou autoclavée (BLOCK et col.). Mais des techniques plus nuancées que l'analyse chimique, à savoir le dosage par la lysine-décarboxylase ou la microbiologie quantitative, ont montré que cette lysine, bien que présente, ne pouvait plus être testée et semblait en quelque sorte masquée. Elle est devenue indisponible et ainsi a perdu tout rôle métabolique. Nous avons déjà mentionné les raisons proposées pour expliquer ce phéno- 
mène: le chauffage fait apparaitre de nouvelles liaisons peptidiques résistantes à 1'hydrolyse enzymatique, rendant la lysine inutilisable. C'est à une hypothèse voisine que font appel HENRY et col. pour expliquer 1'altération de lait sec conservé à $40^{\circ}:$ : les modifications semblent dues, en partie, à une réaction de MAILLARD entre les groupes aldéhydiques des sucres réducteurs et certains groupes aminés libres, parmi lesquels on sait que figurent largement les groupes $\Sigma$-aminés de la lysine $\%$.

\section{Les fourrages deshydratés}

La dessication artificielle des fourrages, notamment de la luzerne, est un sujet à l'ordre du jour. On trouve dans la littérature de nombreux renseignements d'ordre technique et économique sur 1'appareillage (séchoirs électriques, à infra-rouge etc...), mais relativement peu de données sur la valeur nutritionnelle du procédé. La majorité des expérimentateurs s'est intéressée au sort du carotène et nous avons déjà mentionné, de ce point de vue, qu'une dessication artificielle bien conduite est préférable à l'action lente du soleil. Depuis quelques années cependant, on s'est penché sur l'efficacité protidique. L'ensemble des travaux admet que les traitements thermiques nuisent à cette dernière, mais nous n'avons pu trouver de comparaisons systématiques entre fourrage frais et desséché. Nous ne ferons état que d'un intéressant travail de Zimmermans qui a comparé l'influence des facteurs température et durée sur l'efficacité protidique de la luzerne déshydratée. Voici ses résultats bruts :

\begin{tabular}{|c|c|c|c|}
\hline Traitement & CUD & VB & Efficacité \\
\hline${ }_{130^{\circ} \mathrm{C} / \mathrm{I} 5}$ minutes....................... & & $\overrightarrow{40,25}$ & \\
\hline $13^{\circ} \mathrm{C} / 60$ minutes............. & 65,29 & & $\begin{array}{l}20,89 \\
20,89\end{array}$ \\
\hline ainutes. & $5^{6,35}$ & 26,67 & 15,03 \\
\hline I $70^{\circ} \mathrm{C} / 60$ minutes. & $53,4 \circ$ & 18,42 & 9,84 \\
\hline
\end{tabular}

On admet que l'efficacité protidique se chiffre par $\frac{\mathrm{CUD} \times \mathrm{VB}}{\mathrm{IOO}}$. Elle est abaissée à la fois par l'intensité du chauffage et par sa durée. Jouant sur ces deux paramètres, $1^{\prime}$ auteur pense que l'élévation de $\mathrm{I}^{\circ} \mathrm{C}$ dans une échelle allant de $130^{\circ}$ à $170^{\circ}$ est beaucoup plus néfaste qu'une augmentation de la durée de chauffe de I minute dans une échelle allant de I5 à 60 minutes: soit, comme terme de base, une efficacité de 27,47 $\left(130^{\circ} / 15\right.$ minutes), toute augmentation de $I^{\circ}$ et de $I$ minute détermine une baisse de 0,4048 . Mais, dans cette perte, $70 \mathrm{p}$. Ioo sont imputables au facteur température et $30 \mathrm{p}$. Ioo seulement au facteur durée.

La conclusion qui ressort de cette étude est qu'il vaut mieux dessécher la luzerne, en augmentant la durée du traitement que l'intensité thermique.

Zimmermann n'a pas recherché systématiquement les causes responsables de la diminution de la VB, mais il pense qu'une action sur la cystine, facteur limitant, peut être invoquée. 


\section{Les céréales}

Le comportement des céréales vis-à-vis du chauffage est curieux en ce sens qu'il y a parfois amélioration de la valeur alimentaire, tantôt diminution.

\section{Traitements thermiques neutres ou favorables}

I a cuisson à l'eau diminue un peu la digestibilité, mais augmente parallèlement la VB. Elle n'a donc pas d'effet sur l'efficacité protidique globale.

\begin{tabular}{|c|c|c|}
\hline Aliments & Auteurs & CUD \\
\hline Blé entier cru ... & MORTAN & $\overline{88}$ \\
\hline Blé entier cuit à l'eau........................... & MUKisain el col. & $\begin{array}{l}80 \\
87\end{array}$ \\
\hline 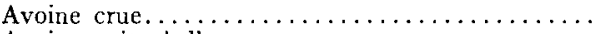 & Mitcinell-Block & 92 \\
\hline Avoine cuite à l'eau $\ldots \ldots \ldots \ldots \ldots \ldots \ldots \ldots \ldots$ & & $9 \mathrm{r}$ \\
\hline Mélange " avoine-maïs-seigle " cru $\ldots \ldots \ldots \ldots \ldots$ & & $9 \mathrm{I}$ \\
\hline Mélange " avoine-maïs-seigle " cuit à l'eau......... & & 89 \\
\hline
\end{tabular}

Le "parching", cuisson rapide du grain dans le sable humide chaud ( 2 à 3 minutes à $130^{\circ}$ ), améliore à la fois 1'utilisation digestive et la Valeur Biologique :

\begin{tabular}{|c|c|c|}
\hline Aliments & Auteurs & CUD \\
\hline Maïs cru $\ldots \ldots \ldots \ldots \ldots \ldots \ldots \ldots \ldots \ldots \ldots$ & ACHARYA et col. & 60 \\
\hline Maïs traité $\ldots \ldots \ldots \ldots \ldots \ldots \ldots \ldots \ldots \ldots \ldots \ldots$ & & 64 \\
\hline Riz cuit à l'eau $\ldots \ldots \ldots \ldots \ldots \ldots \ldots \ldots \ldots$ & & 86 \\
\hline 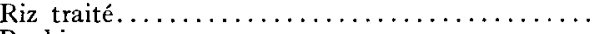 & & 90 \\
\hline Raghi $c r u, \ldots \ldots \ldots \ldots \ldots \ldots \ldots \ldots \ldots$ & & 89 \\
\hline Raghi traité $\ldots \ldots \ldots \ldots \ldots \ldots \ldots \ldots \ldots \ldots$ & & 93 \\
\hline Juar ${ }^{\prime} r u, \ldots \ldots \ldots \ldots \ldots \ldots \ldots \ldots \ldots \ldots \ldots$ & & 83 \\
\hline Juar traité $\ldots \ldots \ldots \ldots \ldots \ldots \ldots \ldots \ldots \ldots \ldots \ldots$ & & 97 \\
\hline
\end{tabular}

La cuisson rapide en autoclave ( 5 minutes à $\mathrm{r}_{2} \mathrm{O}^{\circ}$ ) suivie d'un bref cylindrage renforce 1'efficacité protidique des céréales. RoUSIER accorde au blé ainsi traité une plus-value de $25 \mathrm{p}$. Ioo par rapport à la farine boulangère. MATET et col. enregistrent les mêmes résultats avec leurs flocons de blé. De même une technique décrite par STEWARD valorise significativement l'avoine.

Le "parching" améliorant la digestibilité, on comprend son effet sur l'efficacité protidique. Les choses sont plus subtiles dans le cas des flocons, c'est-à-dire des céréales autoclavées-cylindrées. En effet, RouSIER, comme MATET, enregistrent des CUD identiques avec le blé cru ou floconné. Le fait est à rapprocher des observations faites avec les produits soumis à l'ultra-mouture. Celle-ci améliore nettement le rendement alimentaire, sans bénéfice particulier pour la digestibilité (JACQUOT). On admet alors que 1'éclatement des membranes cellulosiques permet aux sucs digestifs d'attaquer le contenu de certaines cellules qui, comme dans 1'assise protéique, est enfermé dans un petit coffre-fort infracturable. On sait en effet que les monogastriques excrètent en nature l'assise protéique du grain ou du son, alors que celle-ci est parfaitement assimilée après éclatement des parois cellulaires. Le bénéfice de l'éclatement est 
donc qualitatif et non quantitatif. I1 porte sur la Valeur Biologique et non sur le taux d'utilisation digestive. De fait, GuILLEMET et Jacguot ont montré que la rétention azotée ou phosphorée était très supérieure dans un produit désintégré sur abrasif que dans une farine meunière ordinaire. Admettons donc la même hypothèse en ce qui regarde les flocons obtenus par procédé thermique : leur efficacité est accrue parce que leur structure interne est détruite et rompues les parois cellulosiques. Traitement thermique et ultra-mouture auraient donc ici la même signification nutritionnelle, à savoir la libération de métabolites nobles, inutilisables dans les céréales en nature.

\section{Traitements thermiques défavorables}

I orsqu'un traitement thermique s'avère favorable aux céréales, nous pensons donc qu'il s'agit plutôt d'un phénomène secondaire (la rupture des parois internes) que de l'action propre de la chaleur. En effet, tout porte à croire que celle-ci est préjudiciable à la valeur protidique des céréales. C'est ainsi que l'application de chaleur sèche en vue de griller, biscuiter ou torréfier, conduit à une perte d'efficacité. Il est bien connu que la croûte du pain est un moins bon aliment azoté que la mie (PFANNESTIEL, et Salomon). De même, les observations de Morgan soulignent l'infériorité des céréales chauffées à sec:

\begin{tabular}{|c|c|}
\hline Aliments & $\begin{array}{l}\text { Gain pondéral du Rat } \\
\text { ( } \mathrm{g} / \mathrm{g} \text { protide ingéré) }\end{array}$ \\
\hline$\longrightarrow$. & - \\
\hline Pain blanc entier.............. & 1,50 \\
\hline 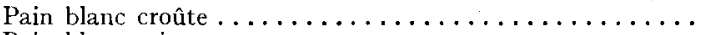 & 0,10 \\
\hline Pain blanc mie $\ldots \ldots \ldots \ldots \ldots \ldots \ldots \ldots \ldots \ldots$ & 1,52 \\
\hline Pain blanc grillé $\left(5_{5} 0^{\circ}\right) \ldots \ldots \ldots \ldots \ldots \ldots \ldots$ & 1,02 \\
\hline 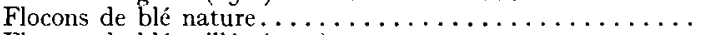 & 1,68 \\
\hline Flocons de blé grillés $\left(200^{\circ}\right) \ldots \ldots \ldots \ldots \ldots \ldots \ldots \ldots$ & I, 12 \\
\hline 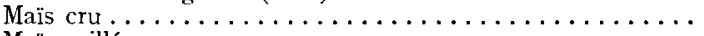 & 1,20 \\
\hline Maïs grillé $\ldots \ldots \ldots \ldots \ldots \ldots \ldots \ldots \ldots \ldots \ldots \ldots$ & 0,82 \\
\hline 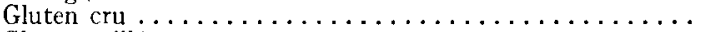 & I,39 \\
\hline Gluten grillé. . . $\ldots \ldots \ldots \ldots \ldots \ldots \ldots \ldots \ldots \ldots \ldots$ & 0,93 \\
\hline
\end{tabular}

La perte d'efficacité de croissance correspond à une double diminution des CUD et de la VB, ainsi que le montrent les valeurs suivantes:

\begin{tabular}{|c|c|}
\hline Aliments & CUD \\
\hline$\cdots$ & - \\
\hline Blé entier cru............... & 88 \\
\hline Blé entier grillé $\ldots \ldots \ldots \ldots \ldots \ldots \ldots \ldots \ldots$ & 73 \\
\hline Gluten cru $\ldots \ldots \ldots \ldots \ldots \ldots \ldots \ldots \ldots$ & 95 \\
\hline 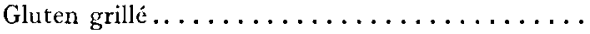 & 90 \\
\hline
\end{tabular}

MATET et col. ont établi le parallélisme entre perte de l'aptitude à la croissance et intensité du traitement thermique :
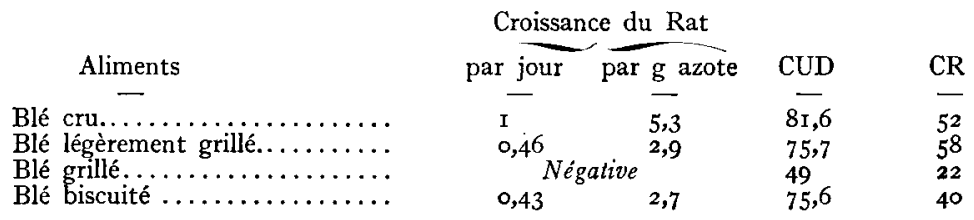
De toutes les opérations industrielles, la plus nuisible semble être celle de l'expansion. La préparation des produits expansés, c'est-à-dire, gonflés ou souflés, comporte une cuisson sous très forte pression suivie d'une décompression brutale. Les spécialités, dites "à breakfast", qui en résultent présentent des aptitudes protidiques très amoindries. D'après STEWARD et col., une céréale "gun expoded" a une efficacité diminuée de $70 \mathrm{p}$. IOO, une avoine " puffed » a perdu $80 \mathrm{p}$. Ioo de ses qualités azotées. Les résultats de MurLin et col. sur blé "inflated ou puffed" sont tout aussi éloquents. Une étude de MITCHELL et BLOCK apporte des précisions sur l'effet propre de chaque stade industriel:

\begin{tabular}{lcc} 
Mélange " avoine-mais-seigle " non traité $\ldots \ldots \ldots \ldots \ldots \ldots$ & CUD & VB \\
Mélange " avoine-mais-seigle " après cuisson $\ldots \ldots \ldots \ldots \ldots$ & 92 & $\frac{63}{63}$ \\
Mélange " avoine-mais-seigle " après expansion $\ldots \ldots \ldots \ldots \ldots$ & 89 & 62 \\
\hline I
\end{tabular}

La seule cuisson diminue la digestibilité sans altérer la VB, alors que l'expansion (gun explosion) provoque un nouvel abaissement des CUD et diminue considérablement la VB.

La conclusion pratique est qu'il est loisible d'utiliser certains types de cuisson industrielle des céréales en vue de préparer des aliments spéciaux comme les "calf and chick starters". Mais on devra choisir des traitements relativement modérés comme la mise en flocons et écarter les techniques brutales de biscuitage ou d'expansion.

La perte d'efficacité des céréales grillées ou expansées s'accompagne d'une baisse significative de la digestibilité et de la VB. Il semble que les protides de céréales se comportent vis-à-vis de la chaleur comme la caséine: bien que le dosage chimique ne montre aucune modification notable, tel ou tel aminoacide serait rendu indisponible. Une expérience de Brock et col. permet de penser qu'il s'agit toujours de la lysine. Le biscuitage fait perdre au "cake " toute aptitude à la croissance du Rat, or la supplémentation par la lysine restaure la valeur protidique initiale.

\section{Les haricots}

Le haricot cru ne possède qu'une très médiocre valeur protidique : administrée comme unique source azotée du régime, cette légumineuse ne permet ni la croissance, ni l'entretien du Rat et détermine des chutes de poids rapidement mortelles (Mc Collum et col.; JoHns et Finks ; JACQUO'T et Rousier etc...). Le fait est patent pour toutes les espèces botaniques du genre Phaseolus: vulgaris, lunatus, angularis etc... On le retrouve également pour Vigna sinensis ou Dolique mongette. L'application de chaleur améliore ici le rendement alimentaire ; mais il convient de noter qu'une simple cuisson, au sens ménager du terme, ne suffit pas pour développer le maximum de valeur alimentaire. En effet, TERroIne 
et VALLA écrivent : "nous n'avons jamais réussi à donner au Porcelet une alimentation dans laquelle la farine de haricot bien cuite, seule source protéique, introduisait $8 \mathrm{~g}$ d'azote par jour, sans provoquer à très bref délai de graves troubles digestifs ". JACQuOT et RousIER ont fait la même observation avec le Rat. Au contraire une application de chaleur industrielle, comme l'autoclavage suivi de cylindrage, améliore considérablement l'efficacité protidique et le haricot ainsi traité, ou haricot éclaté, devient un bon aliment azoté. C'est ce qui ressort des expériences de JACQUOT et RousIER utilisant la technique des régimes alternés :

\section{Régime}

Farine de Haric

Farine de Haricot cuite...........

Farine de Haricot cuite ............... Variation quotidienne CUD Bilan brut d'azote
du poids du Rat

$\begin{array}{lll}- & - & - \\ -0,6 & \overline{1} & +\overline{473} \\ +\mathrm{I}, 5 & 70 & 33\end{array}$

On voit que tout le bénéfice porte sur la digestibilité : c'est la mauvaise utilisation digestive du haricot cru qui conduit à un déficit d'azote. La valeur azotée de cette légumineuse est ainsi limitée par deux facteurs : une carence en aminoacides soufrés qui peut être corrigée par la supplémentation, une résistance des protides à l'hydrolyse enzymatique qui peut être neutralisée par un traitement thermique judicieux. Des recherches in vitro ont d'ailleurs confirmé que la phaséoline isolée, principal constituant azoté des haricots, n'est pas digestible à l'état cru, mais le devient après chauffage. Voici quelques observations de WATERMAN et JoHns sur ce point:

Phaséoline

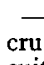

cuite.
$\mathrm{N}$ digéré/ $\mathrm{N}$ total (pepsine-trypsine)

28

$4 \mathrm{I}$

En résumé : la médiocre utilisation digestive des protides de haricot à l'état cru entraîne des bilans azotés négatifs qui, à eux seuls, justifient les pertes de poids vif. Un chauffage du type de celui utilisé pour l'obtention des produits éclatés permet 1'attaque diastasique des protéines de haricot et leur bonne assimilation. Ce bénéfice pourrait vraisemblablement s'interpréter par une dénaturation portant sur les ponts soufrés et la transformation des groupes SS en groupes SH. A côté de ce phénomène général, il faut rappeler que certains haricots contiennent des poisons thermolabiles et sont détoxiqués par la chaleur. C'est le cas pour Phaseolus lunatus qui renferme des quantités parfois élevées d'acide cyanhydrique et pour quelques Stizolobium à dihydroxyphénylalanine.

\section{Les pois}

Comme le haricot, le pois est caractérisé par une déficience en acides aminés soufrés, notamment en méthionine ; mais, contrairement au haricot, le pois ne gagne aucunement a être chauffé. C'est ainsi que l'auto- 
clavage et le blanchîment, qui valorisent considérablement les divers Phaseolus, altèrent l'efficacité protidique de Pisum sativum. Quant au pois éciaté, c'est-à-dire autoclavé et cylindré, il faut utiliser un traitement très ménagé pour respecter l'aptitude alimentaire et tout excès de chauffe est néfaste. Voici, sur ce point, quelques résultats de BLAIsOT et JACQUOT :

Un autoclavage de I heure fait baisser des deux tiers la valeur de croissance du pois pour le Rat ; cet inconvénient disparaît en réduisant le temps d'autoclavage à $\mathrm{I} 5$ minutes. Dans ces conditions le produit naturel n'est pratiquement pas altéré, mais il n'est pas non plus amélioré. Le pois chiche (Cicer arietinum) se comporte vis-à-vis du chauffage industriel exactement comme le pois rond : action insignifiante de l'autoclavage modéré, effondrement de l'aptitude à la croissance pour un autoclavage plus long.

\section{Les lentilles}

Malgré la créance populaire, les lentilles ne représentent qu'un aliment assez médiocre. Elles ont à leur actif une haute teneur en azote ; par contre leur utilisation digestive et leur Valeur Biologique sont relativement basses (Terroine et Valia ; Blaisot ; Strumpeli etc...). Ces défauts, d'ordre qualitatif, sont encore plus manifestes après application de chaleur. Car, malgré les conclusions de Jones et MurPhy, nous estimons avec BLAISOT que les techniques usuelles d'éclatement rendent la lentille complètement inapte à la croissance du Rat. Comme avec les différentes espèces de pois, on peut, en réduisant la durée et la pression de l'autoclavage, obtenir les avantages pratiques des aliments tout-cuits sans diminuer sensiblement la valeur nutritive, mais, même ainsi, la digestibilité azotée est abaissée. On peut donc sauvegarder au maximum les qualités de l'aliment naturel, mais on ne saurait les améliorer par le chauffage.

Ainsi, trois types de légumes, de même origine botanique, de même désignation diététique, à savoir les féculents, se comportent très différemment vis-à-vis des traitements thermiques: le haricot seul est nettement amélioré par une application de chaleur industrielle, alors que les pois ou les lentilles, traités de même façon, accusent une efficacité sensiblement diminuée.

\section{Les traitements thermiques liés à l'extraction des huiles}

Jusqu'ici les traitements thermiques des aliments étudiés présentaient un caractère subsidiaire : le chauffage ne constituait pas une nécessité, mais une simple modalité pratique. Généralement il avait pour but de réaliser des produits prêts à l'emploi. Nous abordons une nouvelle 
face du problème avec l'étude des tourteaux. Ici l'application de chaleur est parfois une nécessité. Ce n'est pas délibérément que certains tourteaux sont cuits, mais par le fait même des modalités d'extraction de 1'huile. Celles-ci se rattachent en gros à deux types de techniques: 1'expression des graines pour expulser 1'huile qu'elles contiennent, l'utilisation des solvants comme véhicule d'extraction. La pression continue ne s'accompagne que d'un faible traitement thermique : les graines sont bien maintenues chaudes, mais leur température ne dépasse pas 60 à $80^{\circ}$. Au contraire, la pression discontinue développe des températures de $1^{\prime}$ 'ordre de $125^{\circ}$. De même, 1'extraction discontinue ne cuit pas le tourteau, alors que l'extraction continue correspond à un véritable traitement thermique, les graines étant portées à $100-120^{\circ}$ pendant une heure au moins. Il s'en suit que selon l'équipement de l'huilerie, l'utilisateur disposera de tourteaux, soit peu chauffés, soit littéralement cuits. Il y a donc ici un intérêt manifeste à étudier l'influence thermique sur le rendement alimentaire.

\section{Le soja}

A 1'état cru, la farine délipidée de soja est, non seulement un aliment très médiocre, mais aussi un aliment dangereux. Elle s'avère inapte à permettre la croissance et cela chez tous les Monogastriques chez lesquels on l'a expérimentée : le Rat (Osborne et Mendel; Mc Collum ; MitCheli, et col. ; Vestal, et col. etc...), le Porc (Robinson; Vestal, et col. ; Shremsbury et col.), le Poulet (Haymard et col. ; Almquist et col. ; MATET et col.).

Cette inaptitude du soja cru va de pair avec l'apparition de signes pathologiques: goîtres accentués (WILGUS et col.), lésions hépatiques (MATET et col.). En un mot: croissance défectueuse, troubles du métabolisme de l'iode, accidents hépatiques accompagnent l'ingestion de soja cru, du moins chez les monogastriques. Tous ces inconvénients disparaissent après chauffage approprié. Nous ne voulons pas multiplier ici les données numériques que 1'on trouvera dans le texte de JACQUOT, MATET et FrIDENSON. Néanmoins, les observations d'Everson et col. méritent d'être citées :

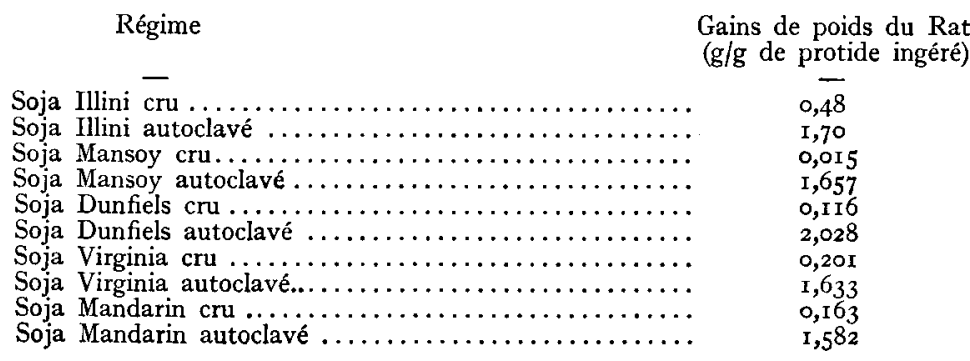


Certains traitements thermiques améliorent donc considérablement l'efficacité du soja et lui confèrent les qualités d'un bon aliment azoté, chez lequel la qualité protidique le dispute à la richesse protidique. Mais pour obtenir ce résultat, il semble que la présence d'eau soit nécessaire. Le chauffage à sec dans une étuve ne détermine qu'une amélioration insignifiante de l'ordre de 25 p. Ivo; un chauffage de même intensité et de même durée en présence de ro p. Ioo d'eau environ (soja chauffé dans un récipient fermé lui conservant son humidité initiale) provoque un bénéfice de 360 p. roo; en présence d'un excès d'eau (autoclavage), ce bénéfice est de plus de 500 p. Ioo. I'intensité du traitement compte autant que sa nature, ainsi qu'il ressort du travail poursuivi sur Poussin par Evans et McGinnis :

\begin{tabular}{|c|c|c|}
\hline & \multicolumn{2}{|c|}{ Gain de poids moyen $(\mathrm{g})$} \\
\hline Régime & en 4 semaines & parg protides \\
\hline Soja cru $\ldots \ldots \ldots \ldots \ldots \ldots \ldots \ldots \ldots \ldots$ & 76 & $\mathrm{I}, 24$ \\
\hline Soja autoclavé 30 minutes à $100^{\circ} \ldots \ldots \ldots$ & I 74 & $\mathrm{I}, 8 \mathbf{i}$ \\
\hline Soja autoclavé 30 minutes à $110^{\circ}$ & 156 & $I, 76$ \\
\hline Soja autoclavé $3 \circ$ minutes à $I 20^{\circ}$. & 162 & $\mathrm{I}, 66$ \\
\hline Soja autoclavé 30 minutes à $\mathrm{I} 30^{\circ} \ldots \ldots \ldots$ & I 20 & $\mathrm{I}, 57$ \\
\hline Soja autoclavé 60 minutes à $130^{\circ} \ldots \ldots \ldots \ldots$ & 69 & 1,09 \\
\hline
\end{tabular}

On voit que l'influence du chauffage passe par un optimum et que tout excès en durée ou en température devient préjudiciable. Cependant les techniques d'expansion, brutales, mais relativement brèves, sont aussi favorables au soja qu'elles sont néfastes aux céréales (MiTchEL, et col.). Ainsi, le fait est amplement démontré : la chaleur humide appliquée avec un certain ménagement améliore considérablement les qualités du soja. Une telle action présente une importance pratique indéniable. Seuls les tourteaux extraits à haute température et en présence d'eau ont une efficacité protidique satisfaisante. C'est le cas pour les produits traîtés par expeller, ce n'est pas le cas pour les tourteaux de presses marseillaises. C'est le cas pour les tourteaux placés en extracteurs verticaux et épuisés à la vapeur et non celui des tourteaux traités en extracteurs cylindriques rotatifs. Il conviendrait que l'huilerie arrive ici à obtenir directement un tourteau cuit sans avoir besoin de recourir à un traitement thermique ultérieur.

I,e mécanisme physiologique qui explique l'amélioration du soja par la chaleur met à la fois en jeu la digestibilité et la rétention métabolique. Voici quelques valeurs numériques empruntées à différents auteurs, qui expérimentaient sur le Rat:

\begin{tabular}{|c|c|c|}
\hline Régime & Auteurs & CUD \\
\hline Soja cru. . $\ldots \ldots \ldots \ldots \ldots \ldots \ldots \ldots \ldots$ & HAYWARD et col. & 85 \\
\hline 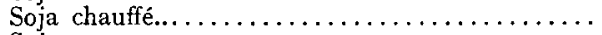 & & 87 \\
\hline 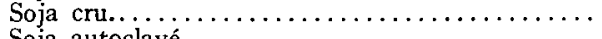 & Mitchell et col. & 78 \\
\hline 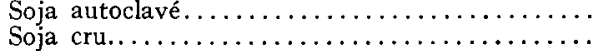 & MELNICK et col. & $\begin{array}{l}83 \\
81\end{array}$ \\
\hline 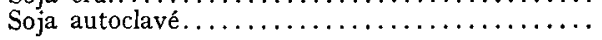 & & 84 \\
\hline
\end{tabular}


L'étude du bilan azoté montre que le bénéfice dû au chauffage porte essentiellement sur la V.B. qui est fortement augmentée alors que la digestibilité n'est que légèrement améliorée. Les mêmes résultats se retrouvent avec le Porc et le Poulet (Wilgus et col. ; TomHave et MuNFORD ; Almquist et col. ; Robinson; Millier et MORrison; Draper et Evans). Ces observations ont été étendues au métabolisme du soufre : la digestibilité soufrée du soja cru et cuit est pratiquement la même (6I et 62 p. IOo), mais la quantité de soufre retenu, c'est-à-dire le Coefficient de Rétention, est bien supérieure avec le soja cuit (25 p. Ioo) qu'avec le soja cru (II p. IOO).

Comment expliquer cette amélioration de la qualité alimentaire par un effet thermique? Plusieurs interprétations ont été suggérées, aucune n'est pleinement satisfaisante.

I ${ }^{\circ}$ Le chauffage détruirait des facteurs inhibiteurs à propriété d'antienzymes. On sait en effet que le soja renferme une substance hydrosoluble thermolabile, douée de propriété anti-trypsique. Ce principe a été étudié sur le plan physico-chimique (HAm et SANDSTEDT; KLOSE et col.) et préparé à l'état cristallisé par KunITz. Ce facteur, inhibiteur de la protéolyse in vitro, serait également inhibiteur de la croissance in vivo. De fait on ralentit considérablement la croissance du Rat ou du Poulet si on surcharge leur régime avec un précipité acétonique d'anti-trypsine. Voici une expérience de Ham et col. :

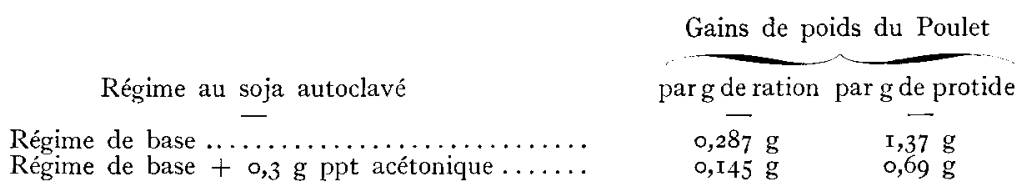

Comme on peut caractériser 1'anti-trypsine dans le contenu intestinal du Poulet alimenté au soja cru, on en déduit: d'une part, que les animaux recevant le facteur anti-trypsique se développent deux fois moins vite que les témoins; d'autre part, que l'inhibiteur se retrouve dans le contenu intestinal.

L'amélioration du soja par la chaleur serait donc imputable à la destruction d'un principe toxique au sens large du terme et non pas à quelque modification de la fraction azotée. A l'appui de cette hypothèse, DÉ et GANGUILY font valoir que la glycine isolée à froid est parfaitement efficace et présente une aptitude à la croissance équivalente, et même supérieure, à celle du soja autoclavé.

Les faits expérimentaux ne sauraient se discuter, aussi doit-on mentionner ceux qui vont à l'encontre de la théorie anti-trypsique. $A$ priori la présence d'un facteur antiprotéolytique devrait influencer 1'utilisation digestive, or nous avons vu que les CUD du soja n'étaient que 
faiblement améliorés par la chaleur, le bénéfice portant surtout sur la VB. D'autre part, il est prouvé que les traitements thermiques ont une répercussion directe sur le contenu protidique de cette graine. Evans et ST-JoHN ont en effet montré que l'autoclavage diminuait le pourcentage des albumines et des globulines, mais augmentait par contre celui des gluténines. Comme ces auteurs ont établi le parallélisme existant entre la VB déterminée sur animal et le taux proportionnel des gluténines, on est porté à croire que l'amélioration observée par action de la chaleur n'est pas uniquement due à la destruction de l'anti-trypsine, mais également à de profondes modifications de la fraction azotée proprement dite. En un mot, la théorie anti-trypsique semble fondée, mais elle n'exclut pas d'autres interprétations.

$2^{\mathrm{o}}$ Le chauffage régulariserait la vitesse d'hydrolyse des aminoacides. Les aminoacides soufrés sont le facteur limitant des protides du soja. La supplémentation par la cystine et surtout par la méthionine est un fait amplement démontré (Mitchels et Smuts ; Hayward et col.; Shrewsbury et Bratzler ; Almouist et col. ; Matet et col. ; MelNICK et col. etc...). De plus les aminoacides soufrés préviennent ou guérissent les accidents provoqués par le soja cru (MaTET et col.). A première vue, les effets de la supplémentation par les acides aminés soufrés semblent donc absolument comparables à ceux que provoque un chauffage approprié. Il est impossible de concevoir que la chaleur puisse provoquer la synthèse de cystine ou de méthionine. D'ailleurs MELNICK et col. ont prouvé que l'autoclavage ne modifiait en rien le taux des aminoacides du soja. Il faut donc admettre une meilleure utilisation des métabolites soufrés, hypothèse d'autant plus engageante que nous avons vu la meilleure rétention du soufre dans le soja chauffé. De plus, Evans et McGinnIs, expérimentant sur Poulet, ont démontré que les traitements thermiques amélioraient notablement les bilans bruts de cystine et de méthionine :

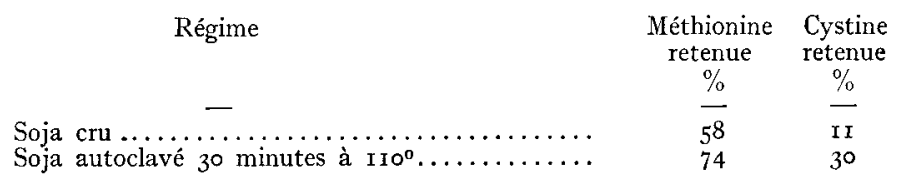

Autrement dit, les acides aminés soufrés seraient indisponibles dans le soja cru et ne pourraient être métabolisés qu'après chauffage. MLINICK et col. se sont efforcés d'expliquer le mécanisme intime de l'effet thermique sur 1a disponibilité de la méthionine. Grâce à une technique d'attaque diasatasique fractionnée, ils ont étudié la cinétique de 1'hydrolyse enzymatique et sont arrivés aux conclusions suivantes : la libération des aminoacides est beaucoup plus rapide après autoclavage du soja; dans le produit cru, la méthionine est libérée beaucoup plus tardivement 
que les autres aminoacides, alors que ce retard disparaît après chauffage. Se basant sur les recherches d'ELMAN qui tendent à prouver la nécessité d'injecter ensemble les indispensables pour obtenir un bilan positif, MELNICK et col. suggèrent que le soja cru manque d'aptitude protidique par le fait que sa méthionine, libérée plus lentement que les autres indispensables, ne peut être métabolisée en même temps que ces derniers. En augmentant le rythme de la libération de la méthionine, le chauffage permettrait qu'elle soit absorbée avec les autres aminoacides. Ainsi, la bonne utilisation des protides alimentaires serait conditionnée, non seulement par la teneur en acides aminés, mais aussi par la vitesse de libération de ces derniers, vitesse qui doit être identique pour tous afin de permettre les supplémentations mutuelles.

Cette hypothèse fort ingénieuse se heurte aux arguments suivants. D'une part, les conditions expérimentales de MELINICK ne ressemblent en rien à celles du travail digestif. Dans son hydrolyse in vitro, le taux de protéolyse du soja est de 8 p. Ioo, alors que les CUD, déterminés dans les mêmes conditions de durée, atteignent 80 p.Ioo. D'autre part, les habitudes alimentaires du Rat et du Poulet cadrent mal avec la théorie. Ces animaux mangent presque continuellement. $\mathrm{Si}$, en raison de sa moindre vitesse d'hydrolyse, la méthionine d'une fraction donnée de nourriture est libérée plus tardivement que les autres aminoacides et risque de manquer d'efficacité, on peut supposer qu'elle retrouve dans la veine porte les aminoacides provenant d'une fraction de nourriture consommée plus tardivement; 1'équilibre est ainsi rétabli et les intersupplémentations peuvent jouer. N'oublions pas, en effet, que les bilans négatifs d'Eiman s'observaient quand un indispensable était injecté 8 heures après les autres : un tel écart est absolument incompatible avec les modes alimentaires du Poulet. Il semble done que 1'hypothèse de Meinick ne soit pas entièrement satisfaisante pour expliquer tous les aspects de l'action bienfaisante de la chaleur sur le soja.

$3^{\circ}$ La chaleur détruirait une albumine à caractère toxique, la soyine. En I952, LIENER et col. ont isolé du soja cru une toxalbumine, la soyine, qu'ils rendent responsable de l'inaptitude alimentaire. Il s'agit, en effet, d'un produit thermolabile. Ils ont pu préparer la soyine à l'état concentré et l'ont expérimentée dans le régime du Rat. L'addition de soyine (I p.IOo) à une ration de soja autoclavé ou de caséine a conduit à un net ralentissement de la vitesse de croissance. Voici les résultats expérimentaux :

Régimes

Gains de poids du rat

Soja autoclavé

(g)

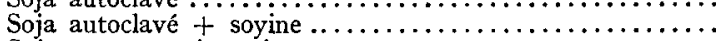

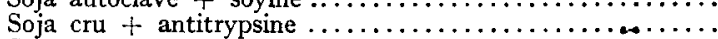

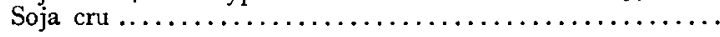

$\overline{66}$

45

49

20 
Pour LIENER deux facteurs seraient donc responsables de la médiocre efficacité protidique du soja cru: la soyine et le facteur anti-trypsique.

Les théories que nous venons d'exposer ne sont pas exclusives l'une de l'autre.

L'essentiel sur le plan pratique est de retenir la valorisation considérable du soja par des traitements thermiques appropriés.

\section{Le coton}

Dans la littérature on trouve pour le coton des Valeurs Biologiques qui varient extraordinairement de 62 à $8 \mathrm{I}$, des Coefficients d'Utilisation Digestive qui vont de 67 à roo. Ces divergences peuvent parfaitement s'expliquer à la lumière de nos connaissances actuelles. Fin effet les protides du coton sont d'autant mieux utilisés qu'ils ont été moins chauffés. Les essais d'Olcot'T et FontaIne, sur Rat sont démonstratifs de ce point de vue:

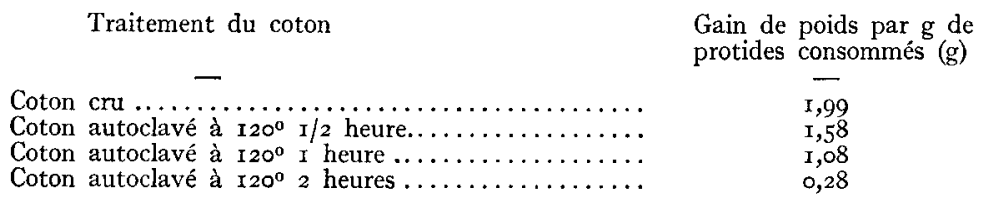

Ici aussi on peut attribuer la perte d'aptitude à la double chute de la digestibilité et de la Valeur Biologique. Les protides du coton ressemblent à la caséine du point de vue sensibilité thermique. Il est vraisemblable que la chaleur rend inutilisable la 1ysine, puisque les farines autoclavées sont efficacement supplémentées par cet aminoacide. Bien que toujours présente, la lysine serait devenue indisponible (inavailable) par suite d'apparition de nouvelles liaisons peptidiques enzymes-résistantes. Quant à l'influence sur la digestibilité, on peut la concevoir comme résultant d'une modification profonde dans la répartition de l'azote. OrcotT et FonTAINe ont, en effet, montré que le chauffage détermine un effondrement de la fraction soluble dans l'eau salée, c'est-à-dire de la somme globulines + albumines + azote résiduel. Celle-ci passe de $75 \mathrm{p}$. Ioo dans le coton cru à I 5 p. Ioo dans le coton cuit à $105^{\circ}$. Autrement dit, la digestibilité semble ici d'autant meilleure que le taux d'azote soluble est plus élevé. La chaleur insolubilisant une partie de l'azote primitivement extractible diminue l'utilisation digestive et par là-même l'efficacité protidique. On peut démontrer la réalité de cette hypothèse par les recherches in vivo, car perte de solubilité et abaissement de la valeur de croissance vont de pair :

\begin{tabular}{cc} 
N soluble dans & $\begin{array}{c}\text { Gain de poids parg } \\
\text { de protides ingérés }(\mathrm{g})\end{array}$ \\
l'eau salée $\%$ & - \\
\hline 45 & $\mathbf{1 , 7}$ \\
38 & $\mathrm{I}, 39$ \\
22 & $\mathrm{r}, 24$ \\
IO & I,1 2
\end{tabular}


Les protides du coton exigent donc d'être le moins chauffés possible pour conserver leur efficacité. Il n'est pas besoin de faire appel au rôle possible du gossypol pour expliquer le phénomène. L'effet thermique joue directement sur la fraction azotée qu'il modifie profondément. Dans la pratique, il faut savoir que les modalités d'extraction des huiles qui conviennent le mieux au soja seraient à proscrire pour le coton.

\section{L'arachide}

Ce tourteau est en France le plus populaire et sa vogue est des plus légitimes. Bien que le problème n'ait pas été étudié systématiquement, il semble bien que ses protides soient indifférents à l'action de la chaleur, du moins dans les conditions de travail des graines oléagineuses. D'une part la cuisson, qui seule permet la digestibilité de la phaséoline, n'améliore aucunement celle de l'arachine (JONES et WATERMAN), d'autre part les essais de MrTCHELL ou ceux de MATET ont montré que des traitements aussi brutaux que le grillage à sec à $150^{\circ}$ n'altéraient pas l'efficacité protidique de l'arachide. On peut en conclure que les techniques de l'Industrie des Oléagineux n'ont aucune répercussion sur la valeur du tourteau résiduaire, du moins sur le plan thermique, car la préparation de tourteaux blancs et dépelliculés est un autre problème.

\section{Le tournesol}

Les études de Mitcheld, et col., de Grau et Almquist, de Matet et col., ainsi que celle toute récente de RoMBAUTs, mettent toutes l'accent sur la remarquable valeur protidique du tournesol. C'est en fait le meilleur aliment azoté d'origine végétale et cela tant en raison de sa richesse protidique que du bon équilibre de ses aminoacides. Aussi est-il intéressant de noter qu'il demeure absolument indifférent à l'action de la chaleur. Il ne risque pas d'être altéré par les extractions à haute température, mais il ne demande pas non plus l'intervention du chauffage pour acquérir son maximum de rendement alimentaire. C'est là un double avantage sur le plan pratique, car l'utilisateur trouve ainsi une qualité standard de tourteau, alors qu'avec le coton ou le soja l'efficacité dépend essentiellement des modalités d'extraction de 1'huile. L'indifférence du tournesol vis-à-vis des traitements thermiques, pressentie par MITCHELL, ainsi que par JACQUOT et col., a été pleinement démontrée par RoMBAUTS. Voici les résultats de cet auteur:

Traitement du tournesol
Gains de poids du Rat par $\mathrm{g}$ de protides ingérés $(g)$

Farine extraite aux solvants

2,0

Farine extraite aux solvants autoclavée ${ }_{1} 5$ minutes à $120^{\circ} \ldots \ldots$.

Farine extraite aux solvants autoclavée 30 minutes à $120^{\circ} \ldots \ldots$. 
Dans la pratique, il n'y a donc pas à se soucier des températures d'extraction de l'huile de tournesol, par contre, et c'est là une considération différente mais impérieuse, il conviendra de décortiquer au mieux les graines de tournesol pour en retirer le meilleur profit, car les tourteaux pailleux ne sont pas alimentaires en raison de leur énorme surcharge cellulosique.

\section{Le palmiste}

$\mathrm{Si}$, en raison de sa faible teneur azotée, le palmiste ne peut être classé parmi les tourteaux nobles, il n'en constitue pas moins un important aliment du bétail de par son abondance et son prix. On connaît ses qualités appéritives, mais on a tendance à sous-estimer sa valeur alimentaire. Certes, c'est un produit encombré de cellulose et ne renfermant guère plus de protides que les céréales. Mais, d'une part la cellulose du palmiste a un comportement alimentaire tout particulier qui fait exception pour le calcul des équivalents fourragers ; d'autre partl'azote du palmiste est qualitativement intéressant et l'équilibre de ses aminoacides lui assigne une place convenable dans la hiérarchie végétale. Malakar et Rombauts lui attribuent une $\mathrm{VB}$ de 65 pour un régime à Io $\mathrm{p}$. Ioo de protides et de $5^{6}$ pour un régime à $\mathrm{I} 3 \mathrm{p}$. Ioo de protides. C'est là un chiffre relativement élevé et on peut dire que le palmiste renferme un azote de bonne qualité, s'il n'en contient pas beaucoup. C'est pourquoi son indifférence vis-à-vis des traitements thermiques mérite d'être soulignée. Elle a été démontrée par MaLAKar et RoMBAUTS, dont voici les résultats :

\begin{tabular}{|c|c|}
\hline Régime au taux protidique de I $2 \%$ & $\begin{array}{l}\text { Gains de poids par Rat } \\
\text { et par jour }\end{array}$ \\
\hline Caséine (témoins) & 2,63 \\
\hline Palmiste $c r u \ldots \ldots \ldots \ldots \ldots \ldots \ldots$ & 2,09 \\
\hline Palmiste autoclavé ${ }_{5}$ minutes à ${ }^{2} 20^{\circ} \ldots \ldots \ldots \ldots \ldots$ & 2,00 \\
\hline Palmiste autoclavé $3^{\circ}$ minutes à $120^{\circ} \ldots \ldots \ldots \ldots \ldots$ & 2,09 \\
\hline
\end{tabular}

On doit en déduire sur le plan pratique que les graisses du palmiste peuvent être extraites par n'importe quel procédé sans avantage, ni dommage pour le tourteau.

\section{Le coprah}

Ce tourteau est plus estimé que le palmiste, bien qu'il ne renferme guère plus d'azote. Sa valeur alimentaire n'a d'ailleurs fait l'objet que de rares investigations. De plus, les résultats sont assez peu concordants à première vue. Ainsi MITCHEL, et VILLEGAs assignent à ses protides une VB de 58 , alors que SmuTs et MALAN trouvent une VB de 69 et MrTCHELL et col., de 76 . Ce dernier chiffre est absolument remarquable pour un aliment végétal. Comme il a été obtenu avec une farine délipidée à froid, on pouvait penser que le coprah est d'autant plus efficace qu'il est 
moins chauffé. C'est 1'hypothèse formulée par MitcheLL, et col. et qu'à titre provisoire avaient retenue JAcQuot, MateT et Firidenson. Depuis, les recherches de ROCHE et BAUDOIN ont mis l'accent sur la variabilité de la méthionine dans les tourteaux de coprah. Alors que les protides des noix fraîches traitées au laboratoire contiennent de I,7 à I, 9 p. roo de méthionine, ce taux tombe à 0,8 dans certains tourteaux commerciaux et même un échantillon d'origine inconnue n'en contient plus que des traces. Il est évident qu'un tel appauvrissement en méthionine s'accompagne d'une diminution notable de la valeur alimentaire. RochE, et BAUDOIN pensent que la chute de la méthionine a pour origine, soit des conditions de conservation défectueuse du tourteau terminé, soit la nature du traitement industriel lui-même. Cette conclusion rejoint donc notre propre hypothèse, $\grave{a}$ savoir qu'il faut chauffer le moins possible la pulpe de noix de coco au cours de l'extraction des graisses pour conserver au tourtean sa valeur optimale.

\section{RESUME ET CONCLUSIONS}

Les traitements thermiques ont donc une influence absolument imprévisible sur la valeur alimentaire. Tantôt, ils la diminuent nettement, tantôt ils l'augmentent, enfin ils n'accusent parfois aucun effet. Rien ne permet de présager du sens de la réponse et seule l'expérimentation peut renseigner en cette matière. Les variations de la valeur alimentaire globale s'accompagnent à la fois de modifications dans 1'utilisation digestive et dans la rétention métabolique. C'est dire que l'application de chaleur peut changer, non seulement la quantité de nutriments offerte à l'organisme en fin du travail digestif (exprimée par le CUD), mais aussi la qualité des aliments (exprimée par la VB). Là se borne notre connaissance des mécanismes intimes, car aucune théorie ne peut pleinement expliciter la cause profonde des effets thermiques. Celles dont nous avons discuté ont du moins l'avantage de montrer que des faits expérimentaux d'ordre essentiellement pratique peuvent conduire à des hypothèses hautement spéculatives sur les mécanismes nutritionnels.

Pour nous résumer, nous proposons de grouper les aliments en fonction de leur comportement vis-à-vis des traitements thermiques.

\section{Aliments indifiérents à la chaleur}

Qu'ils soient crus ou chauffés, le tournesol, l'arachide et le palmiste conservent une même efficacité protidique. I1 s'agit de sous-produits industriels ; on peut déduire qu'ici les modalités d'extraction de l'huile n'influencent aucunement la valeur du tourteau résiduel du moins sur le plan thermique et que l'utilisateur n'a pas à se soucier de savoir si le tourteau a été cuit ou non. 


\section{Aliments améliorés par la chaleur}

La fécule n'est bien digestible que cuite; la pomme de terre crue doit donc être éliminée de la nourriture du bétail, d'autant plus qu'elle peut provoquer de graves accidents intestinaux. Il y a un avantage certain, non seulement à cuire la pomme de terre, mais à utiliser des flocons ou farines obtenus par traitement industriel. Les protides du haricot sont également indigestibles à l'état cru. Mais, dans ce cas, une simple cuisson au sens " ménager " du terme, semble ne pas suffire et il faut faire appel à la chaleur industrielle (autoclavage et cylindrage) pour tirer le meilleur profit de cette légumineuse. Le soja cru est un bien médiocre aliment azoté, non seulement mal assimilé, mais encore générateur de troubles multiples : goitre et nécroses hépatiques. Après un traitement thermique adapté (application de chaleur humidedans des conditions de température et de durée déterminées), le soja devient un excellent aliment totalement inoffensif. Il conviendrait donc de réserver au soja les méthodes d'extraction d'huile qui comportent une application de chaleur. Il faudrait également que l'utilisateur puisse avoir la garantie relative à la cuisson du tourteau.

\section{Aliments détériorés par la chaleur}

Sont thermosensibles, dans le sens d'une diminution de l'efficacité biologique, les protides du lait, des tissus animaux, des fourrages, des céréales, des pois, des lentilles, du coton, du coprah. Dans ce cas, tout traitement thermique un peu intense a de graves incidences sur le rendement alimentaire. Les Industriels devraient donc se soucier des répercussions de leurs techniques sur le plan nutritionnel. Voici quelques indications dans ce sens. Dans le traitement du coton et du coprah, éviter les procédés qui cuisent la graine. Veiller à toute surchauffe dans la préparation du lait sec ou concentré. Préconiser l'obtention de farines de poisson ou de viande à basse température et sous vide au détriment des produits séchés à la flamme. Se rappeler que, lors de la déshydratation artificielle du fourrage, le facteur température est plus préjudiciable que le facteur temps. Recommander les flocons de céréales obtenus par des traitements doux et s'abstenir des céréales expansées préparées à l'aide de procédés brutaux. Contrôler, afin de les réduire au minimum, la température et la durée de l'autoclavage lors de la préparation des pois ou de lentilles " éclatés "...

Certes, toutes ces recommandations ont leur importance et on ne devrait jamais perdre de vue l'influence possible des traitements thermiques. Il est toujours indiqué de tirer le meilleur profit d'un aliment. 
Cependant, sur le plan strictement pratique, deux observations peuvent en quelque sorte limiter la portée de nos conclusions.

Tout d'abord, les résultats que nous avons présentés intéressent toujours les Monogastriques: Rat, Souris, Porc, Volaille. Ils n'ont certainement pas la même signification pour les Ruminants. I,es exigences des Bovins et des Ovins sont qualitativement beaucoup moins impérieuses que celles du Porc ou du Poulet. Notamment en ce qui regarde le besoin protidique : autant la notion d'indispensabilité et d'équilibre des aminoacides est importante chez les Monogastriques, autant elle est subsidiaire chez les Ruminants. Or, les traitements thermiques ont une répercussion indéniable sur la VB; celle-ci n'ayant qu'une importance secondaire chez les Polygastriques, on peut en déduire que le chauffage ne modifie guère les aptitudes des aliments à satisfaire les besoins de la Vache ou de la Brebis. De fait, la littérature zootechnique, qui parle abondamment de l'influence des traitements thermiques dans le rationnement du Porc ou du Poulet, est muette lorsqu'il s'agit du gros bétail. Par exemple, Terrorne a insisté sur la non digestibilité de la farine de haricot mal cuite pour le Porc, alors que les auteurs anglais préconisent ce produit comme aliment de sevrage du veau, sans aucunement mentionner s'il s'agit du produit cru ou cuit. De même, le soja, qui doit avoir été chauffé pour être efficace dans le régime du Porc et de la Volaille, semble parfaitement utilisable à 1'état cru par le Veau. SkrnNe's et KING n'ont en effet enregistré aucune amélioration du soja par la chaleur en prenant le Veau comme réactif animal. On peut déjà conclure que les effets thermiques, dignes d'intérêt dans la nutrition des Monogastriques, ont nettement moins d'importance dans celle des Ruminants.

Il y a donc là une première restriction quant à la portée générale des faits précédemment décrits. Une autre remarque est tout aussi limitative. I es différences observées après chauffage le furent en " testant " l'aliment, cru ou traité, selon les méthodes de la physiologie, c'est-à-dire en l'utilisant comme unique source azotée du régime. Or, les animaux ne sont pas monophages et leur régime est des plus complexes, surtout en ce qui regarde les matières protidiques. On doit dès lors se souvenir que l'influence de la chaleur - quel qu'en soit le sens - est parfaitement comparable aux effets de la supplémentation. Le soja cru est amélioré aussi bien par cuisson que par addition de méthionine. L'adjonction de lysine aux céréales expansées leur restitue leur efficacité première. Les déductions pratiques sont alors faciles à tirer. Dans les normes de 1'alimentation usuelle, le besoin de cuire le soja est beaucoup moins impérieux si le reste de la ration est assez riche en méthionine. Les inconvénients des céréales trop chauffées sont moins graves si le régime contient par ailleurs beaucoup de lysine. Et ces notions s'appliquent au cas des Monogastriques dont on connaît les exigences toutes particulières. 
En un mot, il faut connaître le sens des effets thermiques, puisqu'il y a toujours intérêt à utiliser un aliment au maximum de son efficacité. Les études de laboratoire sur ce point se justifient donc pleinement. Néanmoins, il faut souligner que leur portée est contingente à la fois des espèces animales et des habitudes alimentaires.

\section{BIBLIOGRAPHIE}

La grande majorité des références bibliographiques se trouve dans les articles de base suivants:

Peterson (W. J.) et Pressi.y (H. B.). - - Influence of cooking processes on food nutrients. A compilation of abstracts. National Cooperative Project, Rep. no VIII, January 1946.

Jacquot (R.), Mate'T (J.) et FRIDenson (O.). - Influence de traitements thermiques industriels sur la valeur protidique des aliments. Ann. Nutrit. Alim., 1947, 1, 157-214.

MELNICK (D.) et OSER (B. L.). - The influence of heat processing on the functionnal and nutritive properties of protein. Food Technology, I949, 3, 57-7I.

Jacquot (R.) et Merat (P.). - Les tourteaux alimentaires, Monographies de l'Iterg, 6I p., Paris, I949.

Creac'H (P.). - Les protides de farine de poisson et leur utilisation dans 1'alimentation animale. Congrès Intern. Poisson, p. 249-294, Paris, I950.

Les références nouvelles sont les suivantes:

ClifCorn (L. E.). -- Advances Food Res., I948, 1, 39-I04.

Zimmermann (G.). - - Trav. Chimie Alim. et Hyg. Suisse, 1946, 37, 35-56.

Zimmermand (G.). - Trav. Chimie Alim. et Hyg. Suisse, I948, 39, I-I9.

Zimmermann (G.). - VII e Congrès Intern. Ind. Agric. Paris, I948, tiré à part.

Guillemet (R.), Jacquot (R.) et Tremolithres (J.). - Ann. Nutrit. Alim., I950, 4, I69-I80.

Sutra (R.). - Bull. Soc. Chim. biol., 1947, 7-8, 738.

LEVY (J.) et JACQUOT (R.). - C. R. Ac. Sc., I948, 22\%, 37I-373.

JACQUOT (R.). - Journ. Recherches Bellevue, 1949, 8, 22I-226.

GuiliLmet (R.) et JAcQuot (R.). - Bull. Soc. Chim. biol., I944, 26, 324332.

Rice (E. E.) et BEuk (J. F.). - Advances in Food Res., I953, 4, 233.

Rombauts (P.). - Oléagineux, I95I, $6^{\mathrm{e}}$ année, 203-2I0 et 275-282.

Roche (J.) et BaudoIN (N.). - Oléagineux, I95I, $6^{\mathrm{e}}$ année, II-I4.

SkinNeiT (J. H.) et King (F. G.). - Ind. Agric. Expt. Sta. Bull. 429, I938. 